\title{
Quantum-mechanical treatment of high-energy channeling radiation
}

\author{
J. Augustin, A. Schäfer, and W. Greiner \\ Institut für Theoretische Physik, Universität Frankfurt, Postfach 11 1932, 60054 Frankfurt-am-Main, Germany
}

(Received 29 December 1993; revised manuscript received 24 October 1994)

\begin{abstract}
An alternative theoretical description of axial electron channeling in the multi-GeV region has been developed. We solve a kinetic equation to evaluate an electron distribution function in axially oriented single crystals. Based on the single-string model, the required matrix elements for radiation and scattering by lattice vibrations are calculated employing solutions of the Dirac equation in cylindrical coordinates. Results obtained for $150-\mathrm{GeV}$ electrons propagating along the $\langle 110\rangle$ axis of germanium are in good agreement with experimental observations.
\end{abstract}

PACS number(s): 61.80.Mk, 41.60. $-\mathrm{m}, 03.65 .-\mathrm{w}$

\section{INTRODUCTION}

Over the past ten years, the interaction of multi-GeV electrons and positrons with oriented single crystals has been investigated with great effort $[1,2]$. In both planar $[3,4]$ and axial channeling experiments [5], in this energy region, one encounters a dramatic enhancement of bremsstrahlung. For a review, see [6]. Electrons aligned to major crystal axes radiate typically most of their kinetic energy when passing through a crystal more than $1 \mathrm{~mm}$ thick. A substantial fraction of this energy loss consists of hard photons and due to relativistic kinematics, the bremsstrahlung quanta are more or less emitted in the channeling direction. These observations led us to question whether axial channeling of ultrarelativistic electrons might be utilized as a new source for twophoton physics [7]. Two electron beams, channeled in opposite directions by the same single crystal, could provide enhanced luminosities for $\gamma-\gamma$ reactions if a sufficiently large number of hard photons were emitted by the electrons and if these photons stayed close to the crystal axes. There is a broad interest in photon-photon physics as can be seen, e.g., from [8]. High-energy photon-photon reactions both with photons backscattered from electron beams and with bremsstrahlung photons, i.e., photons generated by the collective charge of a particle bunch in an accelerator, are sought. We want to determine whether channeling as a source of high-energy photons can compete with these other options.

Channeling electrons stay close to rows or planes of crystal atoms, "sensing" their electric fields throughout the whole passage through the medium. Therefore any reasonable theoretical description of channeling phenomena must go beyond perturbation theory in treating the interaction between electrons and crystal fields. Detailed models of channeling radiation have been developed by Baier, Katkov, and Strakhovenko [9-11] as well as by Kononets and Ryabov [12,13]. Both Baier, Katkov, and Strakhovenko's semiclassical theory and Kononets and Ryabov's model assume channeling radiation to be basically of synchrotron nature and use the constant-field ap- proximation to evaluate radiation probabilities. Although especially Kononets and Ryabov are very successful in describing the data obtained by Medenwaldt et al., [3-5], they use phenomenological constants to incorporate the influence of angular momenta on the transverse electron distribution.

In this paper we choose a more rigorous approach to axial electron channeling. Starting with the simplest version of the continuum description introduced by Lindhard [14], i.e., the single-string model, the Dirac equation is solved in cylindrical coordinates. These wave functions are employed to calculate radiation and scattering matrix elements, which enter a rate equation for evaluating the electron distribution function. All information about the channeled electrons and the emission of radiation quanta is contained in this distribution. Since we start from first principles, we are certain not to miss any large relativistic effects such as those connected with, e.g., virtual pairs created in the extremely strong channeling fields. For many years we have studied the properties of overcritical electric fields [15]. Ultrarelativistic channeling is often classified as a similar phenomenon. By solving the Dirac equation we can check whether it really involves nontrivial quantum effects, missed by a semiclassical model. We shall show that this is not the case. To solve the Dirac equation for channeling, considerable numerical problems had to be mastered, which are mainly due to the large effective mass that governs the transverse motion of the channeled particles.

The main objective of this work is to calculate photon production in high-energy channeling, to check whether the quasiclassical results are approximately right, to derive the radial photon distribution, and to decide whether $\gamma-\gamma$ collisions of channeling photons in the 100 $\mathrm{GeV}$ range offer a practicable experimental possibility. Detailed theoretical analyses of various quantummechanical properties at $\mathrm{MeV}$ energies can be found in Ref. [16]. These investigations are very interesting, especially for the use of channeling radiation as a probe in solid-state physics. They include, e.g., a detailed description of the interaction with phonons and show that quantum effects are not in general negligible. 


\section{DIRAC EQUATION AND THE SINGLE-STRING MODEL}

In the single-string model the channeled particles interact with only one row of crystal atoms positioned along a straight line. (The direction defined by this line will be called the $z$ direction throughout this paper.) The electrostatic potential describing this interaction is obtained by averaging the projectile-atom potentials over the $z$ direction. For the Debye-Turner potential [17] one obtains the thermally averaged potential

$$
V_{T}\left(r_{\perp}\right)=-\frac{2 e^{2} a_{0}}{d} \sum_{i=1}^{4} \frac{a_{i}}{B_{i}+2 u_{1}^{2}} \exp \left(-\frac{r_{\perp}^{2}}{B_{i}+2 u_{1}^{2}}\right) \text {. }
$$

This potential represents an analytical approximation to relativistic Hartree-Fock calculations. The numerical values of the constants $a_{i}$ and $B_{i}$ are taken from Ref. [18]. $d$ and $u_{1}$ denote the interatomic distance in the string direction and the one-dimensional mean square amplitude of thermal lattice vibrations, respectively. $a_{0}$ is the Bohr radius and $r_{\perp}$ is the distance perpendicular to the channeling axes.

Inserting the potential (1) into a Dirac equation, relativistic wave functions for axial channeling can be computed (we use natural units $\hbar=c=1$ and the Dirac representation of the $\gamma$ matrices according to Ref. [19]). Since (1) depends only on $r_{\perp}$, the problem has cylindrical symmetry. Fortunately the Dirac equation

$$
\begin{aligned}
& \left\{-i \gamma^{0}\left(\gamma^{1} \cos \varphi+\gamma^{2} \sin \varphi\right) \frac{\partial}{\partial r_{\perp}}\right. \\
& -i \gamma^{0}\left(\gamma^{2} \cos \varphi-\gamma^{1} \sin \varphi\right) \frac{1}{r_{\perp}} \frac{\partial}{\partial \varphi} \\
& \left.-i \gamma^{0} \gamma^{3} \frac{\partial}{\partial z}+\gamma^{0} m+V_{T}\left(r_{\perp}\right)-E\right\} \psi=0
\end{aligned}
$$

can be separated in cylindrical coordinates $r_{1}, \varphi, z$ [20]. Following the separation procedure of Shishkin and Villalba [21], we are led to the wave function

$$
\psi_{E, p_{z}, \mu, s}(\mathrm{r}, t)=\frac{1}{\sqrt{2 \pi}} e^{i\left(p_{z} z-E t\right)} \phi\left(\mathbf{r}_{\perp}\right),
$$

with the four-spinor $\phi\left(\mathbf{r}_{\perp}\right)$ describing the transverse motion

$$
\begin{aligned}
\phi\left(\mathbf{r}_{\perp}\right)= & \frac{1}{\sqrt{8 \pi r_{\perp}}} \frac{1}{\left(p_{z}-i m\right)} \\
& \times\left[\begin{array}{l}
\left(-i p_{z}-m-s m_{\perp}\right) e^{i(\mu-1 / 2) \varphi_{u}} u_{1}\left(r_{\perp}\right) \\
\left(+i p_{z}+m-s m_{\perp}\right) e^{i(\mu+1 / 2) \varphi_{u}} u_{2}\left(r_{\perp}\right) \\
\left(-p_{z}+i m-i s m_{\perp}\right) e^{i(\mu-1 / 2) \varphi_{u}} u_{1}\left(r_{\perp}\right) \\
\left(-p_{z}+i m+i s m_{\perp}\right) e^{i(\mu+1 / 2) \varphi_{u}} u_{2}\left(r_{\perp}\right)
\end{array}\right] .
\end{aligned}
$$

Here $m$ denotes the electron rest mass and $m_{\perp} \equiv \sqrt{p_{z}^{2}+m^{2}}$ the transverse mass. The wave functions $u_{1}\left(r_{\perp}\right)$ and $u_{2}\left(r_{\perp}\right)$ depend on the chosen values for $E$ and $p_{z}$; see also Eq. (4). More precisely, the quantum numbers characterizing such a channeling state are the total energy $E$, the momentum in $z$ direction $p_{z}$, the $z$ component of the total angular momentum $\mu$, and the spin orientation $s= \pm 1$. The spin operator for the cylindrically symmetric Dirac equation (2) that is diagonalized by $\psi_{E, p_{z}, \mu, s}$ has a slightly different form than the usual one:

$$
S=\frac{1}{m_{\perp}}\left[-i \frac{\partial}{\partial z} \gamma^{5}+m \gamma^{3} \gamma^{5}\right]
$$

Since the longitudinal momentum $p_{z}$ is a good quantum number, the transverse energy $\epsilon \equiv E-m_{\perp}$ is a conserved quantity as well. Only states with negative values of $\epsilon$ describe electrons that are bound to the string, i.e., that are channeling. Particles with positive energy $\epsilon$ have transverse momentum and therefore move from one string of crystal atoms to another.

The structure of the equations determining the radial functions $u_{1}\left(r_{\perp}\right)$ and $u_{2}\left(r_{\perp}\right)$ is very similar to the spherically symmetric case:

$$
\begin{aligned}
& {\left[\frac{d}{d r_{\perp}}-\frac{\mu}{r_{\perp}}\right] u_{1}-\left[E+s m_{\perp}-V_{T}\left(r_{\perp}\right)\right] u_{2}=0 .} \\
& {\left[\frac{d}{d r_{\perp}}+\frac{\mu}{r_{\perp}}\right] u_{1}+\left[E-s m_{\perp}+V_{T}\left(r_{\perp}\right)\right] u_{1}=0 .}
\end{aligned}
$$

As already mentioned above, the main effects that cause transitions between different channeling states are emission of radiation and scattering by thermal lattice vibrations. Treating the electromagnetic field $A^{\mu}$ of the emitted photon as a perturbation, the first-order $S$-matrix element for a radiative transition reads

$$
S_{f i}=-i e \int d^{4} x \bar{\psi}_{f} A \psi_{i}
$$

Here $i=\left(E_{i}, p_{z i}, \mu_{i}, s_{i}\right)$ and $f=\left(E_{f}, p_{z f}, \mu_{f}, s_{f}\right)$ represent the quantum numbers of the initial and the final state, respectively. From $S_{f i}$ a double-differential radiation probability per unit length and final electron momentum can be derived:

$$
\begin{aligned}
\frac{d^{2} w_{\mathrm{rad}}}{d z d p_{z f}}=\alpha \frac{m_{1 i}}{p_{z i}}\{ & \left|\lambda_{1}\right|^{2}\left(R_{12}-R_{21}\right)^{2} \\
& +\mid \lambda_{1} \frac{k_{z}}{\omega}\left(R_{12}+R_{21}\right) \\
& \left.+\left.\lambda_{2} \frac{k_{1}}{\omega}\left(R_{11}-R_{22}\right)\right|^{2}\right\} .
\end{aligned}
$$

The energy $\omega=\sqrt{k_{z}^{2}+k_{\perp}^{2}}$ and momentum components of the emitted photon follow from the conservation laws for the initial energy and the momentum in $z$ direction:

$m_{\perp i}+\epsilon_{i}=m_{\perp f}+\epsilon_{f}+\sqrt{k_{z}^{2}+k_{\perp}^{2}}, \quad p_{z i}=p_{z f}+k_{z}$.

The $R_{i j}$ denote the occurring radial integrals $\left(\Delta \mu \equiv \mu_{i}-\mu_{f}\right)$ 


$$
\begin{aligned}
& R_{11}=\int_{0}^{\infty} d r_{\perp} u_{1 f} u_{1 i} J_{\Delta \mu}\left(k_{\perp} r_{\perp}\right), \\
& R_{12}=\int_{0}^{\infty} d r_{\perp} u_{1 f} u_{2 i} J_{\Delta \mu+1}\left(k_{\perp} r_{\perp}\right), \\
& R_{21}=\int_{0}^{\infty} d r_{\perp} u_{2 f} u_{1 i} J_{\Delta \mu-1}\left(k_{\perp} r_{\perp}\right), \\
& R_{22}=\int_{0}^{\infty} d r_{\perp} u_{2 f} u_{2 i} J_{\Delta \mu}\left(k_{\perp} r_{\perp}\right),
\end{aligned}
$$

where $J_{\Delta \mu}\left(k_{\perp} r_{\perp}\right)$ is a Bessel function of the first kind. The coefficients $\lambda_{1,2}$ are combinations of the electronic quantum numbers, whose meaning become transparent if they are expanded into the parameters $m / p_{z i}$ and $m / p_{z f}$ :

$$
\begin{aligned}
\lambda_{1} & =\frac{1}{2}\left\{1+\frac{p_{z i}+i m}{s_{i} m_{\perp i}} \frac{p_{z f}-i m}{s_{f} m_{\perp f}}\right\} \\
& \approx \frac{1}{2}\left\{1+s_{i} s_{f}+i s_{i} s_{f}\left[\frac{m}{p_{z f}}-\frac{m}{p_{z i}}\right]\right\}, \\
\lambda_{2} & =\frac{1}{2}\left\{\frac{p_{z i}+i m}{s_{i} m_{\perp i}}+\frac{p_{z f}-i m}{s_{f} m_{\perp f}}\right\} \\
& \approx \frac{1}{2}\left\{s_{i}+s_{f}+i\left[s_{i} \frac{m}{p_{z i}}-s_{f} \frac{m}{p_{z f}}\right]\right\} .
\end{aligned}
$$

The scattering of channeling electrons by lattice vibrations is also considered in first-order perturbation theory. One can derive the following scattering probability per unit length [22-24]:

$$
\begin{aligned}
\frac{d w_{\text {scat }}}{d z}=d[ & \left\{\left|\left\langle\phi_{f}\left(\mathbf{r}_{\perp}\right)\left|V\left(\mathbf{r}_{\perp}-\mathbf{u}_{\perp}\right)\right| \phi_{i}\left(\mathbf{r}_{\perp}\right)\right\rangle\right|^{2}\right\}_{T} \\
& \left.-\left|\left\langle\phi_{f}\left(\mathbf{r}_{\perp}\right)+V_{T}\left(r_{\perp}\right) \mid \phi_{i}\left(\mathbf{r}_{\perp}\right)\right\rangle\right|^{2}\right] .
\end{aligned}
$$

Again the subscript \{\}$_{T}$ denotes thermal average. Employing the harmonic approximation for the lattice vibrations this averaging assumes the form

$$
V_{T}\left(r_{\perp}\right)=\frac{1}{2 \pi u_{1}^{2}} \int d^{2} u_{\perp} e^{-u_{\perp}^{2} / 2 u_{1}^{2}} V\left(\mathbf{r}_{\perp}-\mathbf{u}_{\perp}\right)
$$

Here $V\left(\mathbf{r}_{\perp}-\mathbf{u}_{\perp}\right)$ is the transverse string potential generated by a charge centered at $\mathbf{u}_{\perp} . V_{T}\left(r_{\perp}\right)$ is the transverse potential resulting from averaging $V\left(\mathbf{r}_{\perp}-\mathbf{u}_{\perp}\right)$ with a Gaussian weight. The charges are assumed to be displaced from the string axes by a typical distance $u_{1}$.

We evaluated Eq. (12) by inserting the transverse wave function (4) and the potential (1) and obtained

$$
\begin{aligned}
\frac{d w_{\text {scat }}}{d z}=\delta_{s_{i} s_{f}} d & {\left[\frac{1}{2 \pi u_{1}^{2}} \int d^{2} u_{\perp} e^{-u_{\perp}^{2} / 2 u_{1}^{2}}\left[\frac{2 e^{2} a_{0}}{d} \sum_{j=1} 4 \frac{a_{j}}{B_{j}} e^{-u_{\perp}^{2} / B_{j}} \mathcal{P}_{j}\left(u_{\perp}\right)\right]^{2}\right.} \\
& \left.-\delta_{\mu_{i} \mu_{f}}\left[\int_{0}^{\infty} d r_{\perp} V_{T}\left(r_{\perp}\right)\left[u_{1 i} u_{1 f}+u_{2 i} u_{2 f}\right]\right]^{2}\right]
\end{aligned}
$$

with $B_{j}$ being the constants introduced in Eq. (1) and $\mathcal{P}_{j}\left(u_{\perp}\right)$ being defined as

$$
\begin{gathered}
\mathcal{P}_{j}\left(u_{\perp}\right)=\int_{0}^{\infty} d r_{\perp} e^{-r_{\perp}^{2} / B_{j}} I_{\Delta \mu}\left[\frac{2 r_{\perp} u_{\perp}}{B_{j}}\right] \\
\times\left[u_{1 i} u_{1 f}+u_{2 i} u_{2 f}\right] .
\end{gathered}
$$

In this equation $I_{\Delta \mu}$ stands for the modified Bessel function. In the derivation of Eq. (14) we again assumed that the crystal atoms perform independent harmonic vibrations. This assumption already entered the evaluation of the channeling potential (1). Collective lattice excitations such as phonons were neglected.

Let us remind the reader that we are interested in checking in this contribution whether relativistic effects such as the presence of virtual particles play an important role in high-energy channeling (i.e., whether highenergy channeling involves overcritical effects or not) and we want to derive an approximate transverse photon distribution. While the incorporation of the coupling to phonons could be important to obtain a high-precision description of the data (see Ref. [16] for the importance of phonons for low-energy channeling), it should not lead to fundamental changes.

In order to correctly describe the channeling process, detailed knowledge about the lattice degrees of freedom is, in general, of secondary importance. Instead a careful determination of both the longitudinal and the transverse energy distributions of the channeled electrons is essential. According to the conservation laws (9), every loss of total energy, i.e., every photon emission, goes along with a transition in the transverse energy of the electron.

With knowledge of the radiation (8) and scattering probabilities (14), the electron distribution function $f(n, z)$ can be computed by means of the rate equation $\left[n \equiv\left(p_{z}, \epsilon, \mu, s\right)\right]$

$$
\begin{aligned}
& \frac{d f(n, z)}{d z}=I_{\text {rad }}+I_{\text {scat }} \text {, } \\
& I_{\mathrm{rad}}=\sum_{n^{\prime}} w_{\mathrm{rad}}\left(n^{\prime} \rightarrow n\right) f\left(n^{\prime}, z\right) \\
& -f(n, z) \sum_{n^{\prime}} w_{\mathrm{rad}}\left(n \rightarrow n^{\prime}\right), \\
& I_{\text {scat }}=\sum_{n^{\prime}} w_{\text {scat }}\left(n^{\prime} \rightarrow n\right) f\left(n^{\prime}, z\right)-f(n, z) \sum_{n^{\prime}} w_{\text {scat }}\left(n \rightarrow n^{\prime}\right) \text {. }
\end{aligned}
$$

Here we have defined the symbolic notation

$$
\begin{aligned}
& w_{\text {rad }}\left(n^{\prime} \rightarrow n\right) \equiv \frac{d^{2} w_{\mathrm{rad}}}{d z d p_{z f}} \Delta p_{z f}, \\
& w_{\text {scat }}\left(n^{\prime} \rightarrow n\right) \equiv \frac{d w_{\text {scat }}}{d z},
\end{aligned}
$$


with $n^{\prime}$ and $n$ denoting the initial and final quantum numbers, respectively. The quantity $f(n, z) \Delta p_{z} \Delta \epsilon \Delta \mu$ is simply the number of electrons at a certain position $z$ in the crystal occupying channeling states in the intervals $\left[p_{z}, p_{z}+\Delta p_{z}\right],[\epsilon, \epsilon+\Delta \epsilon]$, and $[\mu, \mu+\Delta \mu]$. Since any loss of longitudinal energy is associated with photon emission, all information about the produced channeling radiation can be deduced from $f(n, z)$.

\section{NUMERICAL SOLUTION AND RESULTS}

For numerical purposes the continuous quantum number $p_{z}$ and the position variable $z$ have to be discretized. The rate equation (16) then becomes a simple matrix differential equation, which can be solved by elementary methods, provided that the spacing $\Delta z$ is chosen sufficiently small.

The actual numerical problems mentioned in the Introduction arise from the computation of the radiation and scattering probabilities (8) and (14). They are basically caused by the huge difference between the two energy scales, which characterize the high-energy channeling processes considered in this paper. The total energies of the channeled electrons and their transverse masses are in the multi-GeV range, while the potential $V_{T}\left(r_{\perp}\right)$ governing the transverse motion is of the order of a few hundred $\mathrm{eV}$. Therefore one has to deal with large quantum numbers for the spinor (4), i.e., for example, hundreds of nodes for the radial functions. One consequence is the presence of a huge number of matrix elements between the existing channeling states, for each of which integrations over highly oscillatory functions must be performed.

However, it turned out that the WKB energy formula leads to an excellent approximation for the energy eigenvalues of the channeling states defined by Eq. (6). This observation enabled us to develop an efficient and fast code for computing the radial functions $u_{1}$ and $u_{2}$.

As a further simplification one can neglect the spin degree of freedom of the channeling wave functions. For almost all combinations of the electronic quantum numbers, spin-flip transitions are strongly suppressed (cf. the example depicted in Fig. 1). Consequently, an unpolarized beam of channeled electrons cannot be polarized by emitting bremsstrahlung, contrary to a claim by Bagrov, Ternov, and Kholomai [25] several years ago.

The following observation is essential for overcoming the remaining numerical problems. The scattering and radiation probabilities can be neglected for most combinations of initial and final quantum numbers. Only in certain limited areas of this parameter space they assume significant values. Furthermore, the radiation probabilities are slowly varying functions in these areas if averaged over a few neighboring final states (cf. Fig. 2). The problem of computing a huge number of matrix elements can therefore be reduced to evaluate a few representative ones.

The strong fluctuations are due to the fact that for fixed (oscillating) functions $u_{1 i}\left(r_{\perp}\right)$ and $u_{2 i}\left(r_{\perp}\right)$, the (oscillating) functions $u_{1 f}\left(r_{\perp}\right)$ and $u_{2 f}\left(r_{\perp}\right)$ change with $\Delta \mu$ and thus for some values of $\Delta \mu$ the integrals in Eqs. (14) and

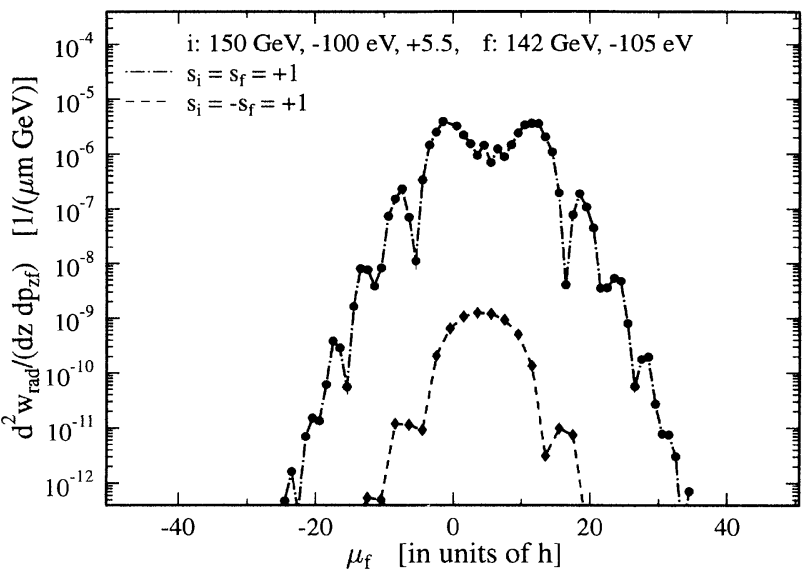

FIG. 1. Photon emission with associated spin flip of the channeled electron is strongly suppressed. This figure demonstrates that axial channeling does not lead to sizeable polarization.

(15) are suppressed by destructive interference. Figures 1 and 2 show the differential rates for individual final states. Such information allows one to obtain the radial profiles in Fig. 5. To obtain the spectrum, one sums over all final states according to Eq. (16).

Figure 3 shows an example for a differential photon spectrum, normalized to one channeled electron. All results discussed in this paper refer to the $\langle 110\rangle$ axis of germanium. Our result is in good agreement with that obtained by Kononets [26]. It is interesting to note that channeling radiation exceeds regular bremsstrahlung due to the Bethe-Heitler process only up to a photon energy of about $75 \%$ of the total electron energy. The reason for the crossing of the curves seems to be simply that because the probability of emitting softer photons is so much higher in channeling, all electrons lose energy much fas-

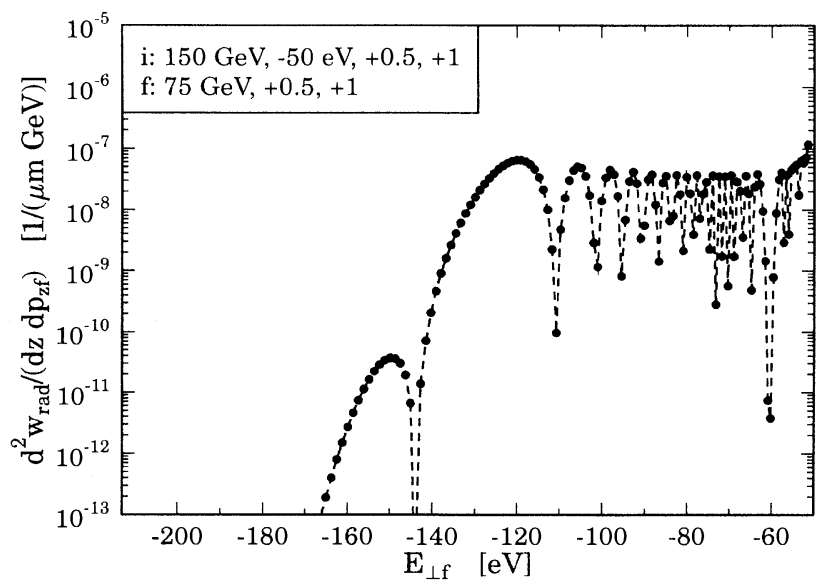

FIG. 2. Averaged over a few neighboring final states, the scattering probability is a slowly varying function of the electron quantum numbers. 


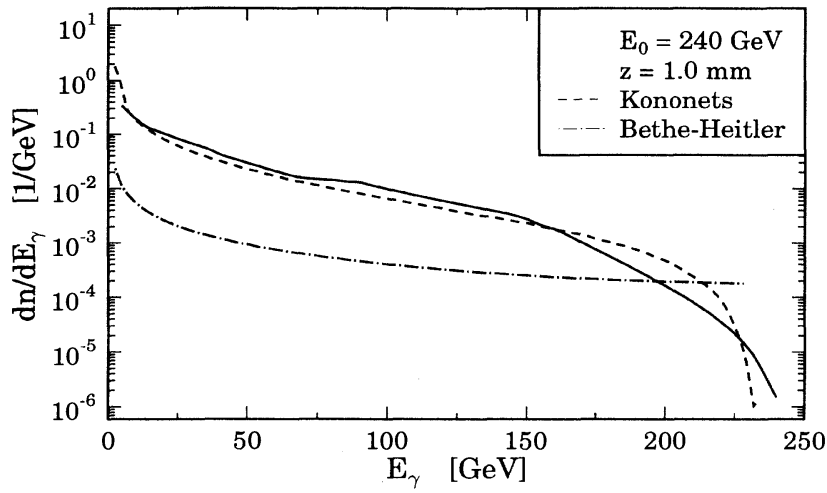

FIG. 3. Photon spectrum compared to a result obtained by Kononets and to the Bethe-Heitler yield. Also plotted is the Bethe-Heitler result for an amorphous target.

ter and therefore have less of a chance to emit photons with energies close to $240 \mathrm{GeV}$ in later stages of their transmission through the crystal.

Angular spatial distributions of photons emitted in axial electron channeling are depicted in Figs. 4 and 5, respectively. Photons with the highest energies are produced at larger distances from the crystal axes than those with lower energies. In addition, emission angles of the order of $10^{-4}$ prevent the photons from staying close to the axis over a macroscopic distance of the order $0.1 \mathrm{~mm}$. Together with the observation of Fig. 3, i.e., photons containing more than $75 \%$ of the initial electron energy are not predominantly produced in channeling experiments, these results indicate that axial channeling of ultrarelativistic electrons cannot serve as an efficient source for high-energy two-photon collisions.

In the remaining figures we compare our results with experimental data taken from [5]. The quantity employed for that purpose is the ratio of the differential energy loss relative to that for an unoriented crystal. For a crystal of length $z$ this is

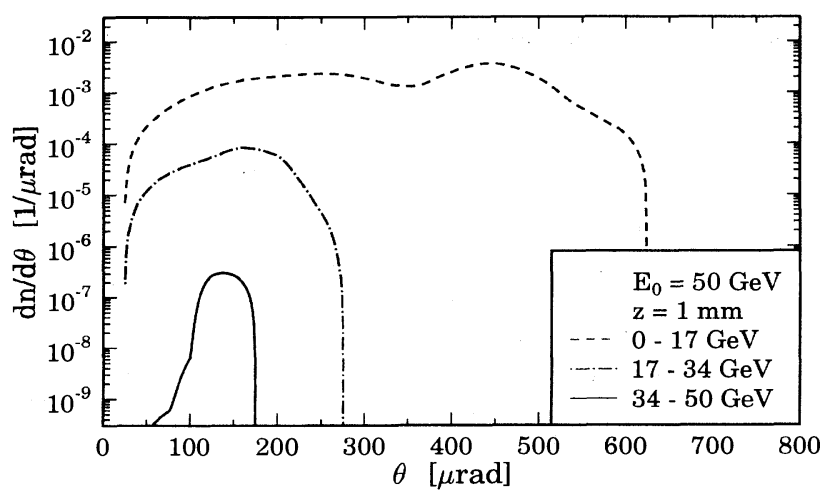

FIG. 4. Angular distribution of photons emitted in axial electron channeling. The polar angle $\theta$ is of the order of $10^{-4}$.

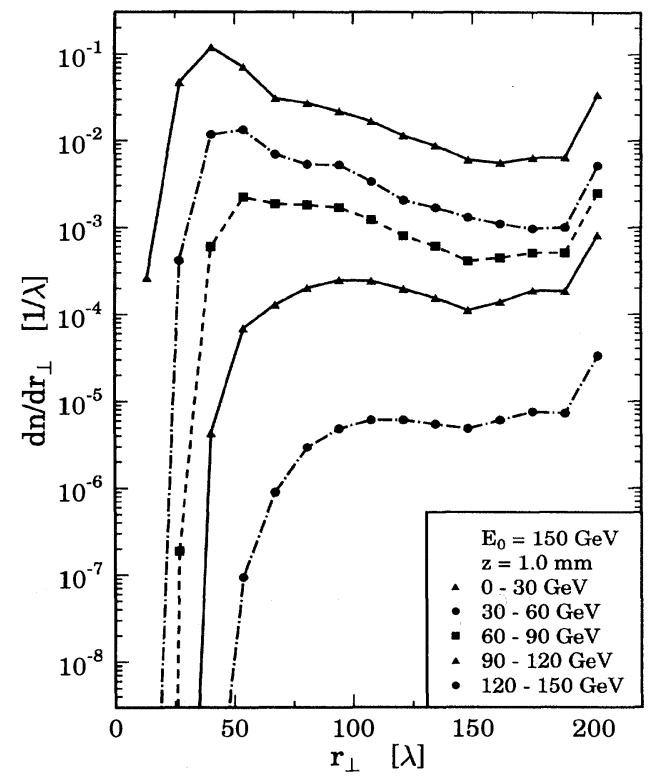

FIG. 5. Spatial photon distribution. Energetic photons are created at larger distances from the crystal axes. Here $\lambda$ denotes the electron Compton wavelength. Obviously the highest-energy photons are produced at rather large distances from the channeling axis.

$$
\text { (enhancement })=E_{\gamma} \frac{d n}{E_{\gamma}}\left(\frac{z}{L_{\mathrm{rad}}}\right)^{-1},
$$

where we have used $z<<L_{\text {rad }}$ and $\exp \left(-z / L_{\text {rad }}\right) \approx 1$ $-z / L_{\mathrm{rad}}$. A charged particle penetrating an amorphous medium of thickness $L_{\text {rad }}$ loses all but a fraction $1 / e$ of its initial energy via regular bremsstrahlung. For electrons of $150 \mathrm{GeV}, L_{\mathrm{rad}}$ assumes values of 93.6 and 23.0 $\mathrm{mm}$ in silicon and germanium, respectively. The energy $E_{\gamma}$ in Eq. (7) is equal to the total radiative energy loss suffered by a channeled electron and not necessarily the

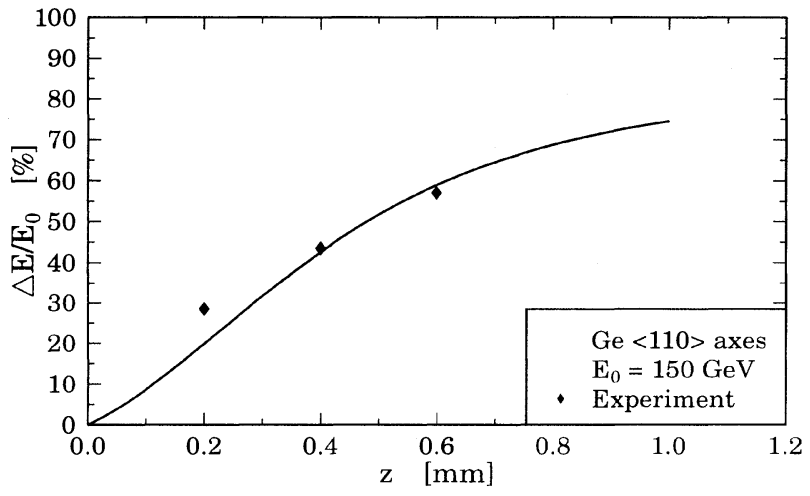

FIG. 6. Total energy loss of $150-\mathrm{GeV}$ electrons channeling along the $\langle 110\rangle$ axis of germanium. The experimental data in Figs. 6-9 are taken from [5]. 


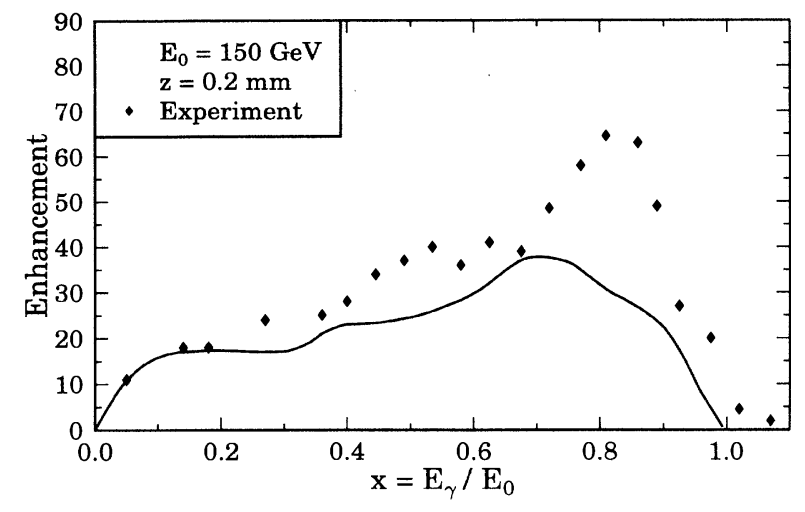

FIG. 7. Differential energy loss for $150-\mathrm{GeV}$ electrons channeled in a germanium crystal. The target thickness is $z=0.2$ $\mathrm{mm}$ and the data are from the same experiment as in Fig. 6; see Ref. [5]. We plotted the differential energy spectrum for a channeled electron divided by the corresponding values for an amorphous target, i.e., the Bethe-Heitler cross section. This enhancement factor is thus dimensionless.

energy of one emitted photon. An electron is more likely to emit several photons during a channeling process, whose individual energies add up to a value $E_{\gamma}$. From our calculations we learn that typically the very large energy losses are on the average due to the emission of four to five photons. Since one has

$$
E_{\gamma} \frac{d n_{\mathrm{BH}}}{E_{\gamma}}=\frac{z}{L_{\mathrm{rad}}}
$$

in amorphous media, the quantity (enhancement) can be understood as the differential radiative energy loss normalized to the corresponding Bethe-Heitler process. One arrives at the total energy loss for the given target thickness plotted in Fig. 6 if the spectra of Figs. 7-9 are integrated. The agreement with the shown experimental data is good enough to prove that our method works in

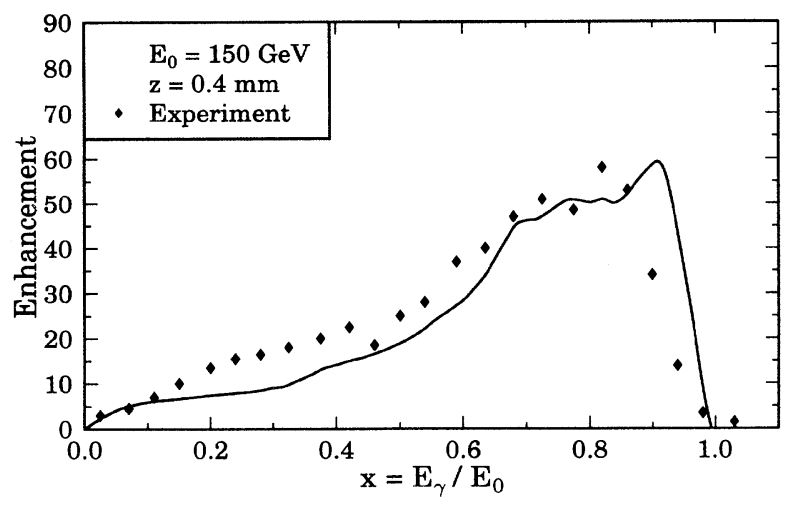

FIG. 8. Same as Fig. 7, but for $z=0.4 \mathrm{~mm}$.

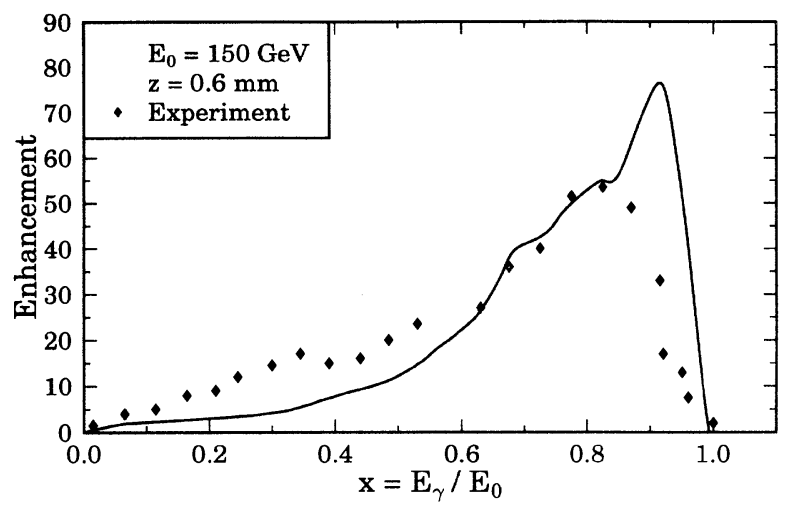

FIG. 9. Same as Fig. 7, but for $z=0.6 \mathrm{~mm}$.

principle. As our results, as well as those obtained with semiclassical methods, describe the data at least approximately, we conclude that quantum effects are not large for these observables. This suggests that the situation in high-energy channeling is qualitatively different from that encountered for overcritical electric fields, but obviously we cannot exclude that the quantum effects are larger for other observables. We want to stress that our calculation is basically parameter free; the only parameters we used characterize the germanium crystal and are well known experimentally.

While the general agreement is rather good, there are clear discrepancies with respect to the $z$ dependence. Our spectra become substantially steeper with increasing $z$, while the experimental slope is more or less constant. We do not understand the origin of this discrepancy. Actual$1 y$, there is a very simple argument why the spectra should become steeper. Just because radiation is so much enhanced, the fraction of electrons that have radiated (usually emitting several photons) most of their energy should become larger and larger with increasing $z$, while the number of electrons with nearly their original energy is suppressed. Because the ratio to the Bethe-Heitler cross section is shown in Figs. 7-9, every enhancement over the Bethe-Heitler cross should lead to such a saturation effect. Further improvement of our results requires intensified numerical efforts, i.e., basically the computation of more representative radiation and scattering matrix elements.

\section{SUMMARY AND CONCLUSIONS}

We developed an alternative description of axial electron channeling in the multi-GeV range. Based on the single-string model and the solutions of the cylindrically symmetric Dirac equation, transitions between the various channeling states due to radiation emission and thermal lattice vibrations have been evaluated. These quantum-mechanical transition probabilities enabled us to determine an electron distribution function, which completely describes the channeling process including the production of photons.

The results presented here show that axial channeling 
experiments can successfully be described by our approach, although the accuracy still calls for improvement. Unfortunately, the idea to utilize axial channeling as a new source for two-photon physics proves to be unrealistic because the photons with high energy are produced rather far out in the string potential. Furthermore, we find that the electrons become only very mar- ginally polarized.

Our model, i.e., solving a rate equation for the distribution function based on quantum-mechanical transition probabilities, should also be applicable to planar channeling. The numerics in that case should even become easier, because the calculation of the transverse wave functions reduces to a one-dimensional problem.
[1] A. Belkacen et al., Phys. Lett. 111A, 86 (1985).

[2] A. Belkacem et al., Phys. Lett. B 1277, 211 (1986).

[3] R. Mendenwaldt et al., Phys. Lett. B 281, 153 (1992).

[4] R. Mendenwaldt et al., Phys. Lett. B 260, 235 (1991).

[5] R. Mendenwaldt et al., Phys. Lett. B 242, 517 (1990).

[6] H. Pilkuhn et al., in Relativistic Channeling, Vol. 165 of NATO Advanced Study Institute, Series B: Physics, edited by R. A. Carrigan and J. A. Ellison (Plenum, New York, 1987), p. 185.

[7] A. Schäfer et al., J. Phys. G 16, L131 (1990).

[8] Photon-Photon Collisions, Proceedings of a Conference on Photon-Photon Collisions, La Jolla 1992, edited by D. O. Caldwell and H. P. Paar (World Scientific, Singapore, 1992).

[9] V. N. Baier, V. M. Katkov, and V. M. Strakhovenko, Zh. Eksp. Teor. Fiz. 92, 1228 (1987) [Sov. Phys. JETP 65, 686 (1987)].

[10] V. N. Baier, V. M. Katkov, and V. M. Strakhovenko, Phys. Lett. A 117, 251 (1986).

[11] V. N. Baier, V. M. Katkov, and W. M. Strakhovenko, Phys. Lett. A 114, 511 (1986).

[12] Yu. V. Kononets, J. Moscow Phys. Soc. 2, 71 (1992).

[13] Yu. V. Kononets and V. A. Ryabov, Nucl. Instrum. Methods B 48, 269 (1990); 48, 274 (1990).
[14] J. Lindhard, K. Dan. Vidensk. Selsk. Mat. Fys. Medd. 34, No. 14 (1965).

[15] W. Greiner, B. Müller, and J. Rafelski, Quantum Electrodynamics of Strong Fields (Springer, Berlin, 1985).

[16] L. V. Hau and J. U. Andersen, Phys. Rev. A 47, 4007 (1993).

[17] P. A. Doyle and P. S. Turner, Acta Crystall. A 24, 390 (1968).

[18] A. H. Sørensen and E. Uggerh øj, Nucl. Sci. Appl. 3, 147 (1989).

[19] C. Itzykson and J.-B. Zuber, Quantum Field Theory (McGraw-Hill, Singapore, 1985).

[20] A. H. Cook, Proc. R. Soc. London Ser. A 383, 247 (1982).

[21] G. V. Shishkin and V. M. Villalba, J. Math. Phys. 30, 2132 (1989); 30, 2373 (1989).

[22] J. U. Andersen et al., Nucl. Instrum. Methods 194, 209 (1982).

[23] J. U. Andersen et al., Phys. Scr. 28, 308 (1983).

[24] J. Augustin, Doctoral thesis, University of Frankfurt, 1993.

[25] V. G. Bagrov, I. M. Ternov, and B. V. Kholomai, Zh. Eksp. Teor. Fiz. 86, 1066 (1984) [Sov. Phys. JETP 59, 622 (1984)].

[26] Yu. V. Kononets (private communication). 